\title{
Receptor-Independent Transfer of Low Density Lipoprotein Cargo to Biomembranes
}

\author{
Markus Axmann, ${ }^{\dagger}$ Erdinc Sezgin, ${ }^{\ddagger}$ Andreas Karner, ${ }^{\S}$ Jiri Novacek, ${ }^{\|}$Michael D. Brodesser, ${ }^{\S}$ \\ Clemens Röhrl, ${ }^{\dagger}$ Johannes Preiner, ${ }^{\S}$ Herbert Stangl, ${ }^{, \dagger}$ and Birgit Plochberger ${ }^{*},{ }_{\odot}$ \\ ${ }^{\dagger}$ Medical University of Vienna, Center for Pathobiochemistry and Genetics, Institute of Medical Chemistry, Vienna 1090, Austria \\ ${ }^{\ddagger}$ MRC Human Immunology Unit, Weatherall Institute of Molecular Medicine, University of Oxford, Oxford OX3 9DS, U.K. \\ ${ }^{\S}$ Upper Austria University of Applied Sciences, Campus Linz, Garnisonstrasse 21, 4020 Linz, Austria \\ "CEITEC, Masaryk University, University Campus Bohunice, Brno 62500, Czech Republic
}

\section{Supporting Information}

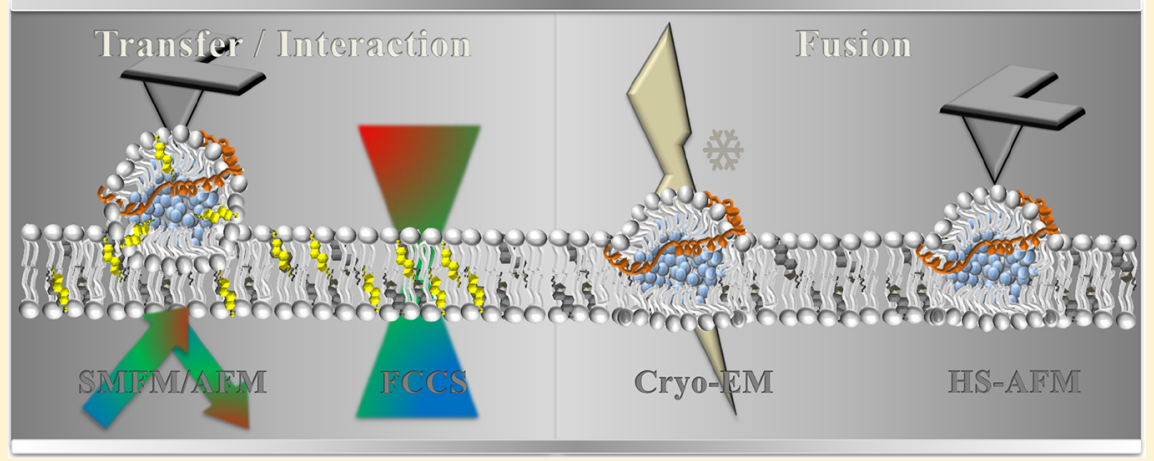

ABSTRACT: The fundamental task of lipoprotein particles is extracellular transport of cholesterol, lipids, and fatty acids. Besides, cholesterol-rich apoB-containing lipoprotein particles (i.e., low density lipoprotein LDL) are key players in progression of atherosclerotic cardiovascular disease and are associated with familial hypercholesterolemia (FH). So far, lipoprotein particle binding to the cell membrane and subsequent cargo transfer is directly linked to the lipoprotein receptors on the target cell surface. However, our observations showed that lipoprotein particle cargo transport takes place even in the absence of the receptor. This finding suggests that an alternative mechanism for lipoprotein-particle/membrane interaction, besides the receptor-mediated one, exists. Here, we combined several complementary biophysical techniques to obtain a comprehensive view on the nonreceptor mediated LDL-particle/membrane. We applied a combination of atomic force and single-moleculesensitive fluorescence microscopy (AFM and SMFM) to investigate the LDL particle interaction with membranes of increasing complexity. We observed direct transfer of fluorescently labeled amphiphilic lipid molecules from LDL particles into the pure lipid bilayer. We further confirmed cargo transfer by fluorescence cross-correlation spectroscopy (FCCS) and spectral imaging of environment-sensitive probes. Moreover, the integration of the LDL particle into the membranes was directly visualized by high-speed atomic force microscopy (HS-AFM) and cryo-electron microscopy (cryo-EM). Overall, our data show that lipoprotein particles are able to incorporate into lipid membranes upon contact to transfer their cargo in the absence of specific receptors.

KEYWORDS: Low density lipoprotein, (high-speed) atomic force microscopy, fluorescence (cross) correlation spectroscopy, single-molecule-sensitive imaging, cryo-electron microscopy, cholesterol transfer

$\mathrm{T}$ ransport of cholesterol in the bloodstream is facilitated by lipoprotein particles, specialized cargo vehicles made of a flexible lipophilic protein scaffold that adapt to different loads of lipid cargo. ${ }^{1}$ These particles are complex conglomerates, assembled by a core unit containing cholesteryl esters and triglycerides, which is enveloped by a shell of free cholesterol, phospholipids, and apolipoproteins. ${ }^{1}$ Besides serving as a structure-lending compound, apolipoproteins affect and regulate the formation of lipoprotein particles and serve as activators or inhibitors of enzymes involved in their metabolism. ${ }^{1}$ In general, lipoprotein particles play a key role in the absorption and assignment of foodborne lipids by the small intestine. Hereby, lipids are transported to the liver and then distributed to peripheral tissues or vice versa (in case of peripheral lipid overload). ${ }^{2}$ The interaction of lipoprotein particles with cell membranes and their uptake is mediated by

Received: January 23, 2019

Revised: February 27, 2019

Published: March 8, 2019 

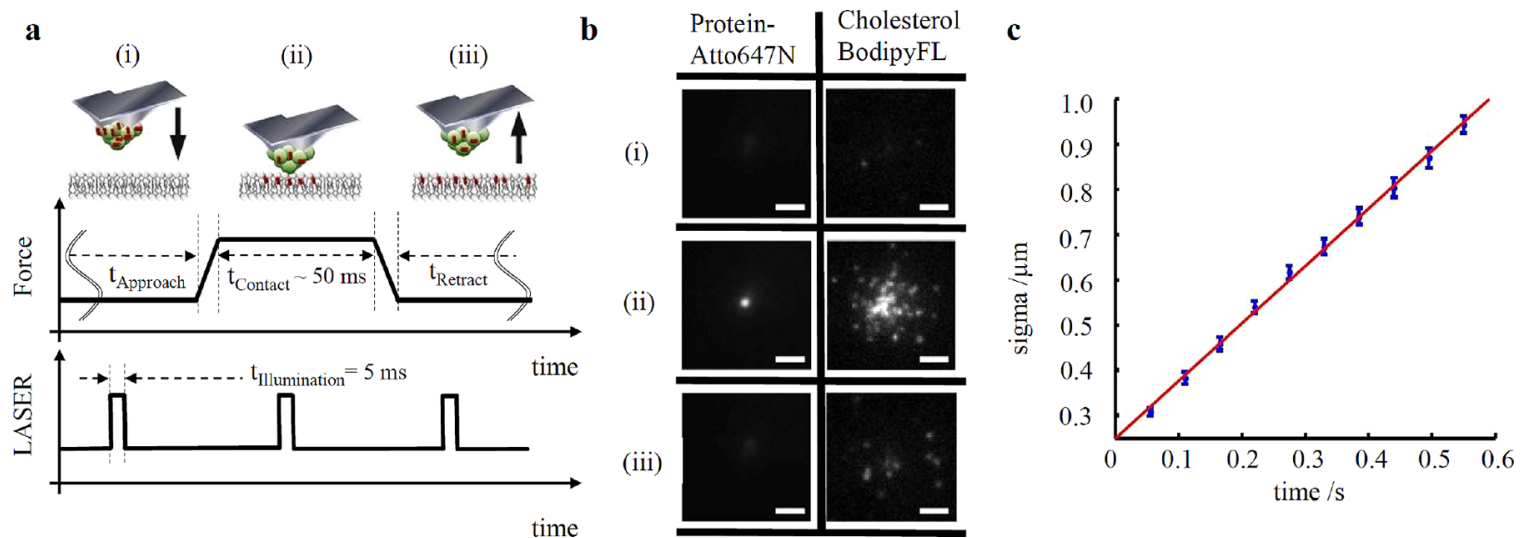

Figure 1. Direct visualization of LDL particle interaction and amphiphilic cargo transfer into the planar supported lipid membrane. With NHSester-Atto647N (for protein-labeling) and cholesterol-BodipyFL bifunctionalized LDL particles were covalently bound to the tip of an AFM cantilever. (a) Sketch of the combined AFM/SMFM approach. The cantilever approached (i) toward the glass supported DOPC bilayer, remained in contact (ii) for a certain time, and was retracted (iii) from the surface. (b) Fluorescence images at the indicated time points are shown for LDL particles preloaded with cholesterol-BodipyFL; as control, Atto647N-labeled apolipoprotein was used. Before contact (i), the fluorescent tip was not observable due to TIR excitation. Transfer of cholesterol-BodipyFL but not of Atto647N-labeled proteins was observable during contact (ii). The tip was retracted (iii) after a contact time of $\sim 50 \mathrm{~ms}$. Measurements $(N=20)$ were performed in PBS at room temperature. SMFM measurements were performed in TIRF configuration. Scale bar $=2 \mu \mathrm{m}$. (c) Transfer of cargo molecules from LDL particles to the bilayer. Flow analysis of 20 contact events yielded a linear increase (red line) of the radial width sigma (see Supporting Information, Single Molecule/Particle Tracking) of the background-corrected fluorescence signal as a function of contact time; $D=0.3 \mu \mathrm{m}^{2} / \mathrm{s}$.

integral membrane receptors. ${ }^{3,4}$ For example, LDL, VLDL, and chylomicron removal from the blood circulation is triggered by binding of the ApoB/E-protein of the lipoprotein particles to the LDL receptor on liver cells, which subsequently clusters in clathrin-coated pits. These pits are afterward endocytosed as vesicles. ${ }^{4}$ The receptor itself unbinds from its cargo in the endosome due to a locally lowered $\mathrm{pH}$ value and is transported back to the cell surface. Individuals with a genetic disorder called familial hypercholesterolemia ( $\mathrm{FH}$ ) have very high levels of circulating LDL particles and thus cholesterol due to mutations in the LDL receptor or the ApoB/E-protein. ${ }^{5,6}$ The LDL receptor is either totally missing (no expression at all or no transport to the cell membrane), unable to bind LDL particles (apoB/E or LDL receptor mutation), or not properly endocytosed/recycled. ${ }^{6}$ Thus, LDL particles and cholesterol are not removed from the blood circulation and cardiovascular disease occurs early in life. Studies on cells from FH-patients and on cells from Watanabe rabbits with heritable hyperlipidaemia (both have defective LDL particle receptors) demonstrated that LDL-particle-mediated cholesterol uptake can take place even in the absence of functional receptors. ${ }^{7,8}$ Thus, direct (i.e., nonreceptor-mediated) cholesterol transfer ${ }^{9}$ has to be a common mechanism for lipoprotein particle interactions. Several molecular dynamic simulation studies supported the anchorage hypothesis, ${ }^{10}$ which in particular implies that distinct hydrophobic and hydrophilic apolipoprotein regions facilitate the engagement to the membrane. Here, we propose that direct cholesterol transfer at the plasma membrane takes place besides the receptor-mediated endocytosis $^{3,11}$ and selective cholesteryl ester uptake. ${ }^{12,13}$

To measure in detail the lipoprotein-particle/membrane interaction and the subsequent cargo transfer, two conditions must be met. First, the cellular system needs to have a controllable complexity and specificity, and second, the observation techniques need to have the required temporal and spatial resolution for visualization of the direct interaction. $^{14-16}$ Owing to the complexity of the cell membrane regarding composition and structure, it is challenging to unequivocally decipher the specific structurefunction relationships and the associated interactions. Thus, in vitro membrane systems are used. These systems allow the tight control of the parameters such as lipid composition, membrane curvature, and membrane tension. ${ }^{17}$ As molecular interactions take place in very fast temporal and small spatial regimes, super-resolving techniques should be applied to obtain information on molecular interactions in combination with well-defined experimental systems. This allows to study interactions at an unprecedented resolution and specificity. $^{18-22}$

We combined several advanced imaging techniques to investigate the interactions of LDL particles with artificial and cell-derived biomembranes. We used planar supported lipid bilayers (SLB), formed on a hydrophilic glass support from pure 2-dioleoyl-sn-glycero-3-phosphocholine (DOPC) vesicles, and performed so-called touch-and-watch experiments. Here, an atomic force microscope (AFM) cantilever tip coated with lipoprotein particles containing fluorescent probes (cholesterol-BodipyFL) served as a nanopipette to deliver molecules to the membrane. Simultaneously, the transfer process was monitored via single-molecule-sensitive fluorescence microscopy (SMFM) (Supporting Information, Movie 1). A sketch of the performed experiment is depicted in Figure 1a: an AFM cantilever-tip coated with fluorescently labeled lipoprotein particles (cholesterol-BodipyFL, protein-bound Atto647N) was moved vertically toward the artificial membrane, brought into contact at a constant force for a certain time, and was subsequently retracted (Figure $1 \mathrm{~b}$ ). Cargo molecules were released into the membrane from LDL particle upon contact without the need for a receptor and exhibit a mobility with a diffusion constant of $D=0.3 \mu \mathrm{m}^{2} / \mathrm{s}$ (Figure 1c).

To avoid any artifacts due to the support of SLBs, we also evaluated the interaction of LDL particles with free-standing giant unilamellar vesicles (GUVs). We first visualized the incorporation of the LDL particles (carrying BodipyFL-labeled cholesterol and Atto647N-labeled proteins) with vesicles using 

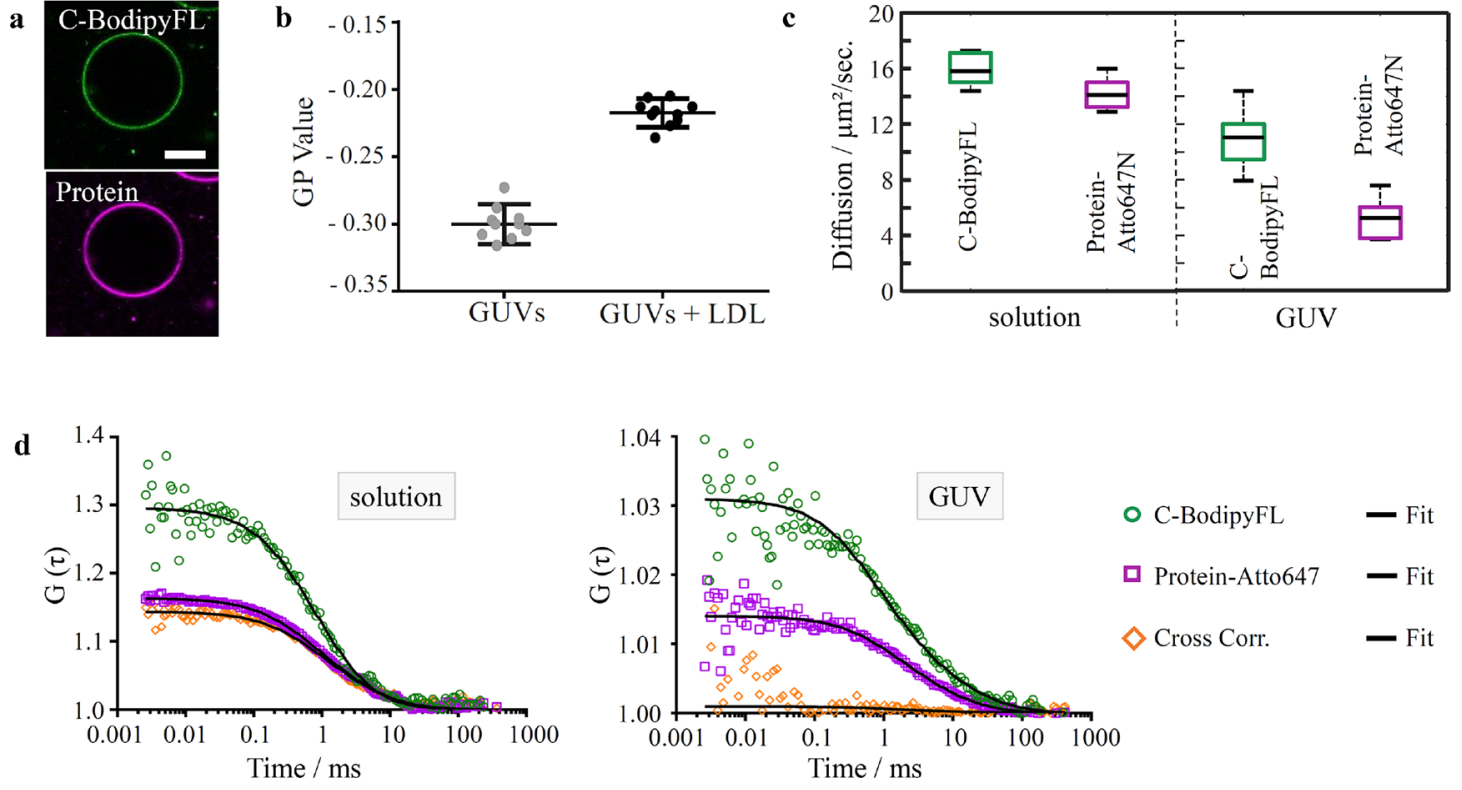

Figure 2. LDL particle induced transfer of amphiphilic cargo into nonsupported biomembranes. FCCS measurements on GUVs incubated with LDL particles. (a) Visualization of LDL interaction and cargo transfer into GUVs for LDL (scale bar $=10 \mu \mathrm{m}$ ). (b) Generalized polarization (GP) value determined with the polarity-sensitive dye C-Laurdan of GUVs alone and LDL blended GUVs represents lipid packing and its hydration level $(N=10)$. (c) Diffusion for C-BodipyFL and protein-Atto647N signal of fluorescently labeled LDL particles in solution and decorated GUVs, respectively. (d) FCS and FCCS on protein-Atto647N and C-BodipyFL. Shown are FCS and FCCS curves for the indicated probe molecules after fusion of LDL particles with GUV membranes (right) or in buffer solution (left). The left graph shows high cross-correlation when LDL particles were intact (magenta and orange curves virtually identical), whereas the graph on the right side shows no notable cross-correlation after LDL fusion with the DOPC membrane. Black lines represent the fits.

confocal microscopy. We clearly observed the incorporation of both cholesterol and proteins into the vesicles (Figure 2a). To confirm that cholesterol is indeed incorporated into the membrane, we measured the lipid packing via the general polarization (GP) analysis of polarity sensitive probe CLaurdan. ${ }^{23}$ Cholesterol is known to rigidify fluid membranes by increasing the lipid packing (or molecular order), thus the incorporation of cholesterol via the lipoprotein fusion should increase the lipid packing of the vesicles. The increase in lipid packing can be quantified using GP analysis; a higher GP value corresponds to a tighter lipid packing. We observed significant increase in GP of GUVs after they were incubated with lipoprotein particles (carrying no fluorescent labels) (Figure 2b). This can be attributed to a reduced membrane fluidity (i.e., high cholesterol content) and thus altered membrane elastic properties (Supporting Information, Figure 1a).

We next performed fluorescence cross-correlation spectroscopy (FCCS) in order to see the molecular interaction between the cargo and particle before and after the lipoprotein particle fusion. FCCS measures codiffusion of fluorescently labeled particles and thus yields information about their interaction. In FCCS, the cross-correlation amplitude changes with codiffusion; it is null when there is no codiffusion and elevated with increasing codiffusion. In solution (i.e., holoparticle), the C-BodipyFL signal strongly overlapped with the protein-Atto647N signal (Supporting Information, Figure 1b). The diffusion coefficients of C-BodipyFL and proteinAtto647N were also similar in solution (Figure 2c). Accordingly, we observed a strong cross-correlation (codiffusion, orange curve) between C-BodipyFL (green curve) and protein-Atto647N (magenta curve) (Figure 2d). In contrast, C-BodipyFL and protein-Atto647N showed no cross-correlation once the particle incorporated itself into GUVs (Figure 2d). Although C-BodipyFL and protein-Atto647N were freely mobile in the GUV membrane, they moved with different diffusion coefficients (Figure 2c), which accounts for lack of cross correlation. This result suggests successful cargo transfer and subsequent separation between the cholesterol and protein fraction of the fused lipoprotein particle.

These observations confirm that LDL particle attachment is sufficient for cargo transfer to model membranes. To test this in more complex and near-native compositions, we prepared giant plasma membrane vesicles (GPMVs) from Chinese hamster ovary $(\mathrm{CHO})$ cells. GPMVs comprise an intermediate biological membrane model system, together with the compositional complexity and protein content of live cell membranes, thus they offer the closest approximation to the cellular plasma membrane. Similar to GUVs, we observed clear fluorescence signal of C-BodipyFL and protein-Atto647 in GPMV membranes (Figure 3a). Moreover, similar to GUVs, cholesterol-transfer from LDL particles alters membrane elasticity of GPMV membranes (Figure 3b)

Furthermore, we applied high-speed AFM (HS-AFM), which allows studying dynamic processes at molecular scale without any labeling, ${ }^{24-26}$ to explore the molecular details of the interaction between LDL particles and biomembranes at a higher spatial (nanometer) resolution. LDL particles were either incubated on a mica-supported DOPC bilayer or immobilized directly on a mica surface. Individual particles were observed as isolated protrusions from the respective surfaces (Figure 4a,b). On mica, we detected spherical LDL particles with an average height of $17.9 \mathrm{~nm}(\mathrm{SD}=3.3 \mathrm{~nm})$ (Figure 4a,c; Supporting Information, Figure 2a,b). On the mica-supported DOPC bilayer, the average height was significantly reduced $(10.1 \mathrm{~nm}, \mathrm{SD}=3.1 \mathrm{~nm}$, Figure $4 \mathrm{~b}, \mathrm{c}$; Supporting Information, Figure $2 \mathrm{a}, \mathrm{b})$ due to particle 

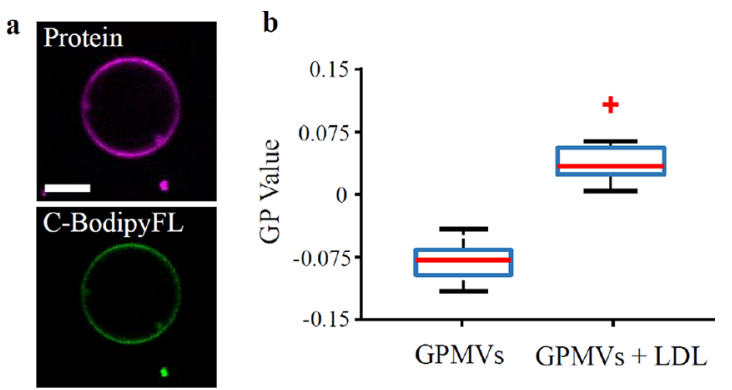

Figure 3. Transfer of amphiphilic cargo from LDL particles to GPMVs increase the GP value. GPMVs incubated with LDL particles. (a) Visualization of LDL interaction and cargo transfer into GPMVs for LDL (scale bar $=5 \mu \mathrm{m}$ ). (b) Generalized polarization (GP) value determined with the polarity-sensitive dye C-Laurdan of GPMVs alone and LDL particles blended GPMVs represents lipid packing and their hydration level $(N=22)$.
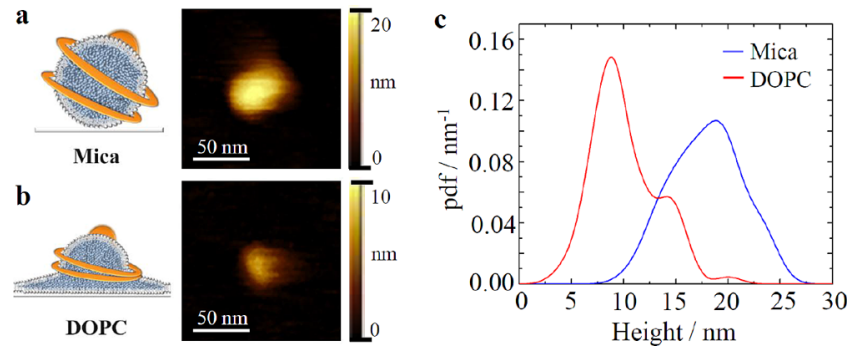

Figure 4. Characterization of LDL particle interaction with a planar, supported lipid membrane and mica. (a) LDL particles incubated on mica and on (b) mica-supported DOPC bilayers were assessed via HS-AFM imaging. (c) Probability density function (pdf) of particle heights on mica (blue line; mean height $=17.9 \mathrm{~nm}, \mathrm{SD}=3.3 \mathrm{~nm}, N=$ 76) and the mica-supported DOPC bilayer (red line; mean height = $10.1 \mathrm{~nm}, \mathrm{SD}=3.1 \mathrm{~nm}, N=225$ ).

integration into the bilayer. LDL particles were immobile on mica; on the mica-supported DOPC bilayer, we were rarely able to observe the time point of particle incorporation (as shown by the sudden particle height drop shown in Supporting Information, Figure $2 \mathrm{c}$ and Movie 2). Interestingly, there were noticeable differences in the interaction behavior of samples from individual donors (compare Figure $4 \mathrm{c}$ and Supporting Information, Figure 2a,b). For all subjects, the height distribution of LDL particles on DOPC membranes (red line) is in general shifted to lower values in comparison to LDL particles on mica (blue line), indicating the partial integration into the lipid bilayer. Thus, we conclude that LDL particles fuse with the supported bilayer membrane upon contact without the need of a receptor similar to HDL particles. ${ }^{27}$ Notably, the interaction behavior strongly varies between donor samples (Figure $4 \mathrm{c}$ and Supporting Information, Figure $2 a, b)$.

As discussed above, HS-AFM data showed that LDL particles have a reduced average height on supported DOPC bilayer. An observation which can either be rationalized via fusion of the particle's outer lipid monolayer with the SBL (height difference approximately equal to the sum of the two displaced membrane leaflets) or a conformational change of the apoB-lipoprotein. To discriminate between these two possibilities, we performed cryo-electron microscopy (cryoEM) experiments with nonsupported membranes (large unilamellar vesicles (LUVs)) mixed with LDL particles (Figure $5 \mathrm{~b}$; Supporting Information, Figure 3a). Owing to their

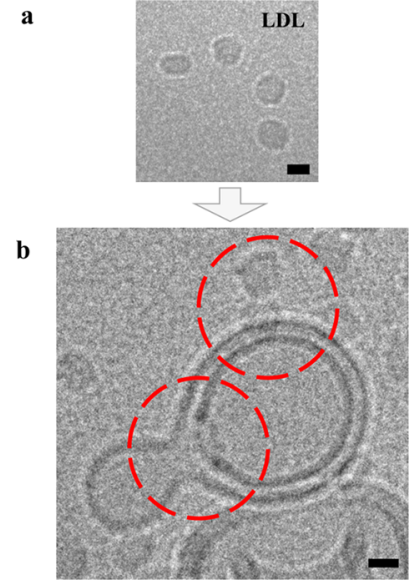

Figure 5. LDL particle fusion with LUVs. Cryo-EM images of (a) single LDL particles and (b) LDL particle decorated LUVs, respectively. Incorporation of LDL particles into the LUV membranes (red circles) was confirmed through recording data under different electron-beam incident angles, thus excluding an accidental overlay of signals originating from different layers of the vitrified ice (see Supporting Information, Figure $3 \mathrm{a}, \mathrm{b})$. Images were acquired under low-dose conditions $\left(20 e^{-} / \AA\right)$ and with the mentioned sample tilt (see Supporting Information, Figure $3 \mathrm{~b}$ ). Scale bar $=10 \mathrm{~nm}$.

different size and morphology, LUVs (Figure 5a; Supporting Information, Figure $3 \mathrm{~b}$, blue arrow) and LDL particles (Supporting Information, Figure 3b, red arrow) can be clearly distinguished. Particle incorporation into the LUV membrane (Supporting Information, Figure 3a, red arrow) was confirmed through recording data under different electron-beam incident angles to exclude a false-positive signal. Thus, we were able to verify general lipoprotein particle fusion with the spherical, nonsupported membrane leaflet of LUVs.

Here, we have investigated the LDL-membrane interaction and the subsequent cargo release to various lipid environments with nanoscopic spatial resolution in real time. The combination of five different techniques allows probing fundamental biological processes at a previously unprecedented level. Regardless of shape, support, and composition of the membrane, lipoprotein particles interact with the membrane without their respective receptor. Direct, receptorless membrane interaction of lipoproteins is a relevant transfer mechanism for amphiphilic cargo to living cells. However, the role of the lipoprotein receptors is ambiguous as the ratio between receptor mediated and direct cargo transfer is unknown and may be influenced by the biocellular state. This study demonstrates that single lipoprotein particles release their cargo through direct contact with membranes, as presented by the touch-and-watch experiment. Even if the particle is not immobilized onto the AFM cantilever tip as shown in our FCCS results and our recent publication, ${ }^{27}$ cargo separates from its transfer vehicle and integrates into the membrane, which is not too surprising in light of the observation of lipid vesicles fusing to membranes. ${ }^{28}$ Interestingly, depending on age and general blood parameters of the sample donor, different behavior in the biomolecular membrane-interaction of LDL particles was observed. As shown in Figure 4 and Supporting Information, Figure 2a,b, our measurements evince different LDL-particle/membrane fusion kinetics. Either the lipid composition is different, especially the cholesterol content, or LDL particles of different donors show alterations, which reduces the interaction. In 
summary, LDL particle fuse with (artificial) membranes as shown in cryo-EM images and transfer cargo, independently of receptor presence or difference in cargo composition. Direct interaction of lipoprotein particles with cellular membranes without the need for a receptor is a relevant transfer mechanism for amphiphilic cargo to and from living cells, which has been recently demonstrated to exist also for HDL particles. $^{27,29}$ This would clarify why cargo molecules on the outer shell of the lipoprotein can be released to cells and, more importantly, how individuals without receptors (FH in humans, ko-mice) are able to survive. These observations reveal a new mechanism for lipid uptake and allow novel insights into how single cell biophysical properties control lipoprotein particle interactions. Because lipoprotein particles interact directly with the membrane, either receptor mediated or simply by fusion and thereby transferring their cargo, they have a high potential as drug carrier systems. These naturally occurring particles could be designed to carry out many beneficial tasks.

\section{ASSOCIATED CONTENT}

\section{S Supporting Information}

The Supporting Information is available free of charge on the ACS Publications website at DOI: 10.1021/acs.nanolett.9b00319.

Experimental methods; supporting figures (PDF)

Movie 1: Combined approach AFMSMFM (AVI)

Movie 2: Temporal interaction of LDL HS-AFM (AVI)

\section{AUTHOR INFORMATION}

\section{Corresponding Authors}

*E-mail: birgit.plochberger@fh-linz.at (B.P.).

*E-mail: herbert.stangl@meduniwien.ac.at (H.S.).

\section{ORCID}

Erdinc Sezgin: 0000-0002-4915-388X

Birgit Plochberger: 0000-0003-2733-9947

\section{Author Contributions}

M.A., E.S., H.S., and B.P. designed the research. M.A., E.S., A.K., J.N., M.D.B., and B.P. conducted experiments. C.R provided chemicals; M.A., E.S., A.K., J.P., M.D.B., and B.P. analyzed data. Supervision, project administration, funding acquisition, and resources: H.S. and B.P. All authors contributed to the writing of the paper. All authors have given approval to the final version of the manuscript.

\section{Notes}

The authors declare no competing financial interest.

\section{ACKNOWLEDGMENTS}

This work has been supported by Austrian Science Fund Project P22838-B13 and P29110-B21, by the Interreg EU project CAC-SuMeR (no. ATCZ14) and the Austrian Research Promotion Agency Innovatives Oberösterreich 2020-851455, by the European Fund for Regional Development (EFRE, IWB 2020), the Federal State of Upper Austria, and "Land OÖ Basisfinanzierung". CIISB research infrastructure project LM2015043 funded by MEYS CR is gratefully acknowledged for the financial support of the measurements at the CF Cryo-Electron Microscopy and Tomography, CEITEC MU. E.S. is funded by Newton-Katip Celebi Institutional Links grant (352333122).

\section{ABBREVIATIONS}

AFM atomic force microscopy; C-BodipyFL cholesterolBodipyFL; cryo-EM cryo-electron microscopy; DOPC 2dioleoyl-sn-glycero-3-phosphocholine; F(C)CS fluorescence (cross)-correlation spectroscopy; FH familial hypercholesterolemia; GPMV giant plasma membrane vesicle; GUV giant unilamellar vesicle; HDL high-density lipoprotein; HS-AFM high-speed atomic force microscopy; LDL low-density lipoprotein; LUV large unilamellar vesicle; SLB supported lipid bilayer; SMFM single-molecule-sensitive fluorescence microscopy; TIRF total internal reflection fluorescence; VLDL verylow density lipoprotein

\section{REFERENCES}

(1) Feingold, K. R.; Grunfeld, C. Introduction to Lipids and Lipoproteins; De Groot, L. J., Chrousos, G., Dungan, K., Eds. MTText.com, Inc., South Dartmouth, MA, 2000; Updated Feb 2 ,2018.

(2) Tall, A. R. An Overview of Reverse Cholesterol Transport. Eur. Heart J. 1998, 19 (Suppl A), A31-A35.

(3) Brown, M. S.; Goldstein, J. L. A Receptor-Mediated Pathway for Cholesterol Homeostasis. Science 1986, 232 (4746), 34-47.

(4) Goldstein, J. L.; Brown, M. S.; Anderson, R. G. W.; Russell, D. W.; Schneider, W. J. Receptor-Mediated Endocytosis: Concepts Emerging from the LDL Receptor System. Annu. Rev. Cell Biol. 1985, 1 (1), 1-39.

(5) Hovingh, G. K.; Goldberg, A. C.; Moriarty, P. M. Managing the Challenging Homozygous Familial Hypercholesterolemia Patient: Academic Insights and Practical Approaches for a Severe Dyslipidemia, a National Lipid Association Masters Summit. J. Clin. Lipidol. 2017, 11 (3), 602-616.

(6) Hobbs, H. H.; Brown, M. S.; Goldstein, J. L. Molecular Genetics of the LDL Receptor Gene in Familial Hypercholesterolemia. Hum. Mutat. 1992, 1 (6), 445-466.

(7) Aliev, G.; Burnstock, G. Watanabe Rabbits with Heritable Hypercholesterolaemia: A Model of Atherosclerosis. Histol. Histopathol. 1998, 13 (3), 797-817.

(8) Edge, S. B.; Hoeg, J. M.; Triche, T.; Schneider, P. D.; Brewer, H. B. Cultured Human Hepatocytes. Evidence for Metabolism of Low Density Lipoproteins by a Pathway Independent of the Classical Low Density Lipoprotein Receptor. J. Biol. Chem. 1986, 261 (8), 38003806.

(9) Zhang, Z.; Lu, L.; Berkowitz, M. L. Energetics of Cholesterol Transfer between Lipid Bilayers. J. Phys. Chem. B 2008, 112 (12), 3807-3811.

(10) Pan, L.; Segrest, J. P. Computational Studies of Plasma Lipoprotein Lipids. Biochim. Biophys. Acta, Biomembr. 2016, 1858 (10), 2401-2420.

(11) Brown, M. S.; Goldstein, J. L. Receptor-Mediated Endocytosis: Insights from the Lipoprotein Receptor System. Proc. Natl. Acad. Sci. U. S. A. 1979, 76 (7), 3330-3337.

(12) Acton, S.; Rigotti, A.; Landschulz, K. T.; Xu, S.; Hobbs, H. H.; Krieger, M. Identification of Scavenger Receptor SR-BI as a High Density Lipoprotein Receptor. Science (Washington, DC, U. S.) 1996, 271 (5248), 518-520.

(13) Meyer, J. M.; Graf, G. A.; Van Der Westhuyzen, D. R. New Developments in Selective Cholesteryl Ester Uptake. Curr. Opin. Lipidol. 2013, 24 (5), 386-392.

(14) Miller, H.; Zhou, Z.; Shepherd, J.; Wollman, A. J. M.; Leake, M. C. Single-Molecule Techniques in Biophysics: A Review of the Progress in Methods and Applications. Rep. Prog. Phys. 2018, 81 (2), 024601.

(15) Elson, E. L.; Fried, E.; Dolbow, J. E.; Genin, G. M. Phase Separation in Biological Membranes: Integration of Theory and Experiment. Annu. Rev. Biophys. 2010, 39, 207-226.

(16) Su, Q. P. L.; Ju, L. A. Biophysical Nanotools for SingleMolecule Dynamics. Biophys. Rev. 2018, 10, 1349-1357. 
(17) Parthasarathy, R.; Yu, C.; Groves, J. T. Curvature-Modulated Phase Separation in Lipid Bilayer Membranes. Langmuir 2006, 22, 5095-5099.

(18) Karner, A.; Nimmervoll, B.; Plochberger, B.; Klotzsch, E.; Horner, A.; Knyazev, D. G.; Kuttner, R.; Winkler, K.; Winter, L.; Siligan, C.; et al. Tuning Membrane Protein Mobility by Confinement into Nanodomains. Nat. Nanotechnol. 2017, 12 (3), 260-266.

(19) Plochberger, B.; Stockner, T.; Chiantia, S.; Brameshuber, M.; Weghuber, J.; Hermetter, A.; Schwille, P.; Schütz, G. J. Cholesterol Slows down the Lateral Mobility of an Oxidized Phospholipid in a Supported Lipid Bilayer. Langmuir 2010, 26 (22), 17322-17329.

(20) Christenson, W.; Yermolenko, I.; Plochberger, B.; CamachoAlanis, F.; Ros, A.; Ugarova, T. P.; Ros, R. Combined Single Cell AFM Manipulation and TIRFM for Probing the Molecular Stability of Multilayer Fibrinogen Matrices. Ultramicroscopy 2014, 136, 211-215.

(21) Sezgin, E.; Schwille, P. Fluorescence Techniques to Study Lipid Dynamics. Cold Spring Harbor Perspect. Biol. 2011, 3, a009803.

(22) Kahya, N.; Scherfeld, D.; Bacia, K.; Poolman, B.; Schwille, P. Probing Lipid Mobility of Raft-Exhibiting Model Membranes by Fluorescence Correlation Spectroscopy. J. Biol. Chem. 2003, 278 (30), 28109-28115.

(23) Sezgin, E.; Sadowski, T.; Simons, K. Measuring Lipid Packing of Model and Cellular Membranes with Environment Sensitive Probes. Langmuir 2014, 30 (27), 8160-8166.

(24) Ando, T.; Kodera, N.; Takai, E.; Maruyama, D.; Saito, K.; Toda, A. A High-Speed Atomic Force Microscope for Studying Biological Macromolecules. Proc. Natl. Acad. Sci. U. S. A. 2001, 98 (22), 12468-12472.

(25) Preiner, J.; Kodera, N.; Tang, J.; Ebner, A.; Brameshuber, M.; Blaas, D.; Gelbmann, N.; Gruber, H. J.; Ando, T.; Hinterdorfer, P. IgGs Are Made for Walking on Bacterial and Viral Surfaces. Nat. Commun. 2014, 5, 4394.

(26) Preiner, J.; Horner, A.; Karner, A.; Ollinger, N.; Siligan, C.; Pohl, P.; Hinterdorfer, P. High-Speed AFM Images of Thermal Motion Provide Stiffness Map of Interfacial Membrane Protein Moieties. Nano Lett. 2015, 15 (1), 759-763.

(27) Plochberger, B.; Axmann, M.; Röhrl, C.; Weghuber, J.; Brameshuber, M.; Rossboth, B. K.; Mayr, S.; Ros, R.; Bittman, R.; Stangl, H.; et al. Direct Observation of Cargo Transfer from HDL Particles to the Plasma Membrane. Atherosclerosis 2018, 277, 53-59.

(28) Cevc, G.; Richardsen, H. Lipid Vesicles and Membrane Fusion. Adv. Drug Delivery Rev. 1999, 38 (3), 207-232.

(29) Plochberger, B.; Röhrl, C.; Preiner, J.; Rankl, C.; Brameshuber, M.; Madl, J.; Bittman, R.; Ros, R.; Sezgin, E.; Eggeling, C.; Hinterdorfer, P.; Stangl, H.; Schütz, G. J. HDL Particles Incorporate into Lipid Bilayers-a Combined AFM and Single Molecule Fluorescence Microscopy Study. Sci. Rep. 2017, 7, 15886. 